\title{
Le score SIAPS, une opportunité de valorisation supplémentaire pour Obésité
}

\author{
L. Genser $\cdot$ M. Pigeyre $\cdot$ J. Dargent $\cdot$ J.-P. Bastard \\ (C) Lavoisier SAS 2016
}

Vous n'êtes pas sans savoir que l'ensemble des revues de formation médicale continue françaises peine à trouver leur place dans la nouvelle nomenclature bibliométrique, en partie depuis l'introduction des points SIGAPS (score individuel d'aptitudes pédagogiques en santé). Ces points sont attribués aux articles indexés dans Medline et sont fonction de l'impact factor de la revue et de l'ordre des auteurs. Le score qui en découle sert d'indicateur pour les candidats aux carrières hospitalo-universitaires. L'incitation à publier dans Obésité parmi d'autres revues françaises s'en trouvait donc doublement limitée faute d'indexation dans Medline et donc d'attribution des précieux points SIGAPS.

Partant de ce constat l'Académie nationale de médecine en soutien au syndicat de la Presse des professionnels de santé a réalisé un communiqué de presse pour la revalorisation des revues médicales en langue française ${ }^{1}$. Désormais les publications pédagogiques dans les revues médicales de langues françaises seront valorisées par le score SIAPS. Ce score validé par la conférence des doyens permet d'évaluer les capacités pédagogiques des candidats aux postes hospitalo-universitaires.

L'attribution de ces points permettra, nous l'espérons, de garantir la survie de ces revues en incitant les auteurs, s'orientant ou non vers ce type de carrière, à montrer leurs capacités didactiques à travers différents formats tout en leur garantissant une valorisation de leur travail et, au-delà, de poursuivre notre devoir de formation médicale continue des médecins francophones.

Dans ce numéro de rentrée, nous vous proposons deux articles consacrés à la thématique des tissukines, un article de synthèse exposant la pharmacologie des antibiotiques chez les sujets obèses et un sur les stratégies de maintien de perte de poids, ainsi qu'un cas clinique illustrant le traitement d'une hernie hiatale tardive par glissement après gastrectomie en manchon. Nous rapportons également les résumés des Journées Benjamin Delessert, qui se sont tenues le 5 février dernier. Enfin, vous retrouverez notre traditionnelle revue de presse.

Bonne lecture et bonne rentrée à tous !

\author{
L. Genser $(\bowtie)$ \\ AP-HP, Service de chirurgie hépato-bilio-pancréatique, \\ transplantation hépatique, Groupe Hospitalier Pitié Salpêtrière, \\ France \\ e-mail : Laurent.genser@gmail.com \\ Institut de Cardiométabolisme et nutrition, \\ Institute of Cardiometabolism and Nutrition, \\ ICAN, Pitié-Salpêtrière Hospital, Paris, 7513, France
}

1. Communiqué de l'Académie nationale de médecine, séance du 26 janvier 2016 sur la valorisation des revues médicales en langue française www.academie-medecine.fr 